\title{
O PROCESSO DE TRABALHO EM UMA UNIDADE BÁSICA NO MUNICÍPIO DO RIO DE JANEIRO
}

\author{
The Process of working in a basic \\ unit in Rio de Janeiro town \\ El Proceso de trabajo en una unidad básica
en la ciudad del Rio de Janeiro
}

Mary Ann Menezes Freire ${ }^{1}$

Fernanda Teles Morais ${ }^{2}$

Beatriz Mancebo D. Albuquerque ${ }^{3}$

Enirtes Caetano Prates Melo ${ }^{4}$

\section{Resumo}

Trata do processo de trabalho dos profissionais de saúde que atuam em uma unidade básica de saúde, em relação ao uso de tecnologias e à rede de relações que se estabelecem na produção do cuidado. Objetivos: Identificar as relações estabelecidas entre os prestadores de cuidado no espaço micropolítico de uma unidade básica; analisar os "ruídos" identificados no processo de trabalho dos profissionais. Método: Metodologia não-convencional, adaptada a um estudo qualitativo. Adotouse um estudo de série de casos, cuja trajetória foi denominada trajetória-sentinela, e, através desta, foram detectados "ruídos" que influenciam na efetividade do cuidado. Conclusão: Foram identificados "ruídos" específicos, intra-institucionais e interpessoais. A análise destes revelou a fragilidade da rede de contratualidade estabelecida no interior dessa unidade, que está diretamente relacionada com o processo de trabalho e com a qualidade da assistência prestada.

Palavras-chave: Saúde Coletiva. Enfermagem. Pessoal de Saúde.

\begin{abstract}
It shows the working process of the health professionals that act in one basic unit of health in relations of the use of technologies and the relationship that establish in the care's production. Objectives: identify the relations established between care's assistance in the micropolitical space of one basic unit; to analyse the "noises" identified in the work's process of the professionals. Method: methodology not conventional adapted to a qualitative study. It was adopted a study of the cases whose trajectory was called trajectory-sentinel and, through that, it was detected "noises" that influenced in the effectivity of the care. Conclusion: it was identified specifics "noises", intrainstitutionals and interpersonals. The analyse of this revealed the fragility of the contractuality's net established in the interior of this unit that is connected directly with the work's process and with the assistence's quality given.
\end{abstract}

Keywords: Public Health. Nursing. Health Personnel.

\section{Resumen}

Se trata del proceso de trabajo de los profesionales de la salud que actúan en una unidad básica de la salud en las relaciones que establecen el cuidado de la producción. Objetivos: identificar las relaciones establecidas entreel equipo de asistencia en el espacio micropolítico de una unidad básica; para analizar los "ruidos" identificadas en el proceso del trabajo de los profesionales. Método: metodología no convencionales adaptados a un estudio cualitativo. Se aprobó un estudio de los casos cuya trayectoria se llama trayectoria-centinela y, a pesar de que, se detectó "ruidos" que influyeron en la efectividad de la atención. Conclusión: se identificaron aspectos específicos "ruidos", intrainstitucionales y interpersonales. El análisis de este puesto se manifiesto la fragilidad de la contratualidade neto establecido en el interior de esta unidad que está conectado directamente con la labor del proceso y con la asistencia de calidad. 


\section{INTRODUÇÃO}

Esta investigação é fruto das experiências de ensino e pesquisa que marcam a parceria entre a Escola de Enfermagem Alfredo Pinto - UNIRIO e uma unidade básica da rede municipal de saúde, situada no Município do Rio de Janeiro. A partir de discussões a respeito do modelo de gestão do cuidado em saúde nesta unidade, aliado a um interesse da equipe e dos gestores em discutir a qualidade da assistência prestada, elegeu-se o setor de pediatria, considerado crítico, como cenário privilegiado para análise da rede de relações que se estabelecem na produção do cuidado.

Investigar a gestão do cuidado em saúde, em geral, não é uma tarefa fácil. Identificar as relações que se estabelecem requer um distanciamento e um tempo nem sempre disponível entre as equipes de trabalho. 0 processo avaliativo deve funcionar como um instrumento de gestão capaz de se desdobrar em intervenções assistenciais que privilegiem a qualidade do cuidado prestado e descrever experiências diversas que agregam obstáculos visíveis e invisíveis. Nesse sentido, identificar o desdobramento das intervenções assistências exige um mergulho no lócus da produção do cuidado.

Os processos de trabalho concretos, possíveis de se vivenciarem nos serviços de saúde, merecem atenção especial na gestão da mudança. Existem "efeitos" do processo de trabalho que se expressam no dia-a-dia dos serviços e que devem ser "olhados" como alvo para operações, que podem disparar potencializações vitais na direção de novos processos de produção do cuidado ${ }^{1}$.

0 cotidiano institucional se expressa como uma dobra (uma quebra) na qual, de um lado, há o cenário do mundo das significações atravessado e não necessariamente compartilhado pelo conjunto dos agentes em situação institucional ${ }^{2}$. Na dobra do sentido e do sem sentido, tanto nos planos individuais como coletivos, existem processos ruidosos operando em cada singularidade, bem como entre elas. Trais processos agem como forças que aparecem "do nada" e se fazem presentes de modo não funcional, causando estranhamentos no cotidiano ${ }^{2}$. 0 cotidiano é o lugar onde há mútuas invasões dos dois mundos entre si. É onde se produzem os estranhamentos, os "ruídos", as "falhas" do mundo com sentido no instituído, e cheio de significados, onde existem e funcionam os acordos e contratos e, ao mesmo tempo, onde os instituintes impõem quebras, linhas de fuga ${ }^{2}$.

Uma análise das interfaces entre os sujeitos instituídos, seus métodos de ação e o modo como se interseccionam permite realizar uma nova compreensão da tecnologia gerencial em saúde. É possível a constituição de tecnologias de gestão do trabalho, que permitam identificar ruídos e quebras ocorridos nos processos de trabalho ${ }^{2}$. Os ruídos representam estranhamentos, problemas no desenvolvimento do processo de trabalho, que poderão interferir ou comprometer a qualidade do cuidado prestado, contradições do processo de trabalho que poderão ser identificados através das ferramentas analisadoras ${ }^{3,4}$.
As tecnologias de gestão do trabalho consistem no uso de instrumentos analisadores e auto-analíticos, como tecnologias potentes para propiciar avaliações do trabalho desenvolvido nos serviços de saúde 2 . Os instrumentos analisadores têm como característica exprimir a problemática de um sujeito, manifestála e denunciá-la ${ }^{5}$, levando em conta as subjetividades circulantes na interação entre os sujeitos envolvidos. 0 fluxograma analisador se constitui num instrumento de análise, que interroga os "para que", os "que" e os "como" dos processos de trabalho, e ao mesmo tempo revela a maneira de governá-lo ${ }^{1}$.

A idéia de tecnologia não está ligada somente a equipamentos tecnológicos, mas também a 'saber fazer' e a um 'ir fazendo'. No campo da saúde, não deve prevalecer a lógica do 'trabalho morto', aquela que se expressa nos equipamentos e saberes estruturados. 0 ser humano necessita das denominadas tecnologias leves 4 .

No dia-a-dia das unidades de saúde observa-se que a disponibilidade de requisitos considerados essenciais como estrutura física em conformidade com padrões técnicos, material permanente e de consumo em quantidade e qualidade satisfatória não são suficientes para garantir a qualidade do cuidado prestado. Há um "algo mais" que agrega as (inter)relações que se estabelecem. 0 estudo teve como objetivo analisar com os profissionais desta unidade básica de saúde o processo de trabalho a partir da análise de casostraçadores.

\section{TRAGETÓRIA METODOLÓGICA}

0 caminho teórico-metodológico adotado nesta investigação foi uma metodologia não-convencional, adaptada a um estudo de natureza qualitativa. Segundo Minayo ${ }^{6}$, essa modalidade de pesquisa, de um modo geral, enfatiza a importância de conhecer, entender e interpretar a natureza das situações e eventos quer sejam eles passados ou presentes. Dessa forma, buscou-se compreender o processo de trabalho dos profissionais de saúde que atuam no setor de pediatria de uma unidade básica de saúde.

0 cenário da pesquisa foi uma unidade básica de saúde localizada no Município do Rio de Janeiro, que equivale ao distrito sanitário. Esta tem sua assistência voltada predominantemente para a área materno-infantil, sendo a pediatria o setor mais demandado pelos usuários. Foram sujeitos da pesquisa 12 profissionais da unidade de saúde que atuam na pediatria ou em setores diretamente ligados à mesma, e 4 mães de crianças menores de seis anos acompanhadas pelo setor de pediatria da unidade investigada.

A pesquisa, que atendeu a resolução de número 196/96, aprovada pelo protocolo de número 38/08, do Comitê de Ética em Pesquisa da Secretaria Municipal de Saúde, que trata de pesquisas envolvendo seres humanos, foi realizada sob termos do consentimento livre e esclarecido.

A memória não-escrita foi resgatada através de entrevistas semi-estruturadas, realizadas com os trabalhadores de saúde envolvidos com o setor de pediatria da unidade e com a mãe da 
criança selecionada. Adicionalmente foram analisados os registros no prontuário da criança. Todo material utilizado (depoimentos, prontuário, registros administrativos) foi acessado exclusivamente para uso dessa pesquisa. 0 estudo não envolveu qualquer tipo de risco, direto ou indireto, para a clientela e para os profissionais envolvidos. Como medidas de confidencialidade dos dados, foram registradas apenas as letras iniciais dos nomes dos respondentes. Vale ressaltar que todos os sujeitos da pesquisa tiveram liberdade de se recusar a participar ou desistir em qualquer fase da pesquisa sem penalização ou prejuízo algum.

A pesquisa foi desenvolvida em duas etapas distintas que compreenderam a configuração de uma rede de pedidos e compromissos marcada pelas contratualidades estabelecidas entre os diversos atores sociais presentes no lócus do cuidado e a avaliação de uma trajetória-sentinela.

Adotou-se um estudo de série de $\operatorname{casos}^{7}$, retrospectivo, utilizando uma trajetória-sentinela que permitiu ordenar os fatos cronologicamente; identificar os serviços envolvidos e as unidades assistenciais utilizadas; localizar os resultados de exames, evoluções e pareceres dos profissionais de saúde e analisar o seu uso, na tomada de decisões. Foram utilizadas duas ferramentas de análise: a rede de petição de compromissos e o fluxograma analíticó.

$\mathrm{Na}$ observação das relações estabelecidas entre os prestadores de cuidado (trabalhador-trabalhador), foi utilizada a rede de petição e compromisso, que permite abrir uma caixa-preta das relações micropolíticas institucionais reveladora, tanto dos tipos efetivos de contratos como dos compromissos estabelecidos, que os vários agentes institucionais em cena realizam entre si, em processo silencioso, muitos dos quais obedecendo a um tipo específico de padrão?

A rede de petição e compromissos, construída através da técnica de grupo focal, permitiu analisar a relação entre 0 serviço da pediatria e os outros serviços/unidades na produção do cuidado a criança. A construção de rede foi considerada completa quando foi identificado o tipo de pedidos e compromissos estabelecidos entre os diversos atores com a pediatria e mesmo internamente ao próprio serviço. Foram considerados elegíveis para participar nesta fase da pesquisa todos os profissionais pertencentes ao setor de pediatria ou de setores que estabelecem relação com a mesma (enfermeiros, nutricionistas farmacêuticos, entre outros).

Para esse primeiro momento do estudo, foi marcada uma data, de acordo com a disponibilidade da chefia da unidade básica, para a realização do grupo focal, onde ocorreram dinâmicas individuais e em grupo. Participaram dessa etapa da pesquisa 12 profissionais da unidade e um moderador que pôde intervir para focalizar e aprofundar a discussão em torno do tema: processo de trabalho na pediatria. Todos os integrantes do grupo focal compartilhavam traços em comum e possuíam uma experiência prévia do assunto abordado ${ }^{8}$.

Para a segunda etapa do estudo foi construído um fluxograma analisador, que consiste em um desenho do modo como está organizado um conjunto de processos de trabalhos, vinculados entre si em torno de certa cadeia de produção?
0 fluxograma, além de representar o que acontece em um serviço de saúde, opera em primeiro plano como um diagramaresumo, que permite esquematizar basicamente todos os processos-chave que ocorrem e caracterizam um determinado serviço de saúde, e que possa servir de guia para a construção dos outros processos nele presente ${ }^{2}$. Esse fluxograma de uma trajetória sentinela permitiu a análise e discussão do modo como opera a rede de (inter)relações no setor estudado.

Nesse tipo de delineamento não cabe a utilização de amostragem probabilística, uma vez que o estudo de um único caso já possibilita a construção do objeto e de suas características essenciais $^{9}$. A trajetória-sentinela, dispositivo analisador da qualidade de assistência, permite avaliar conjuntamente todo processo assistencial e resultados, sinalizando ruídos ao longo do acompanhamento do caso índice, momentos e formas de operacionalização do trabalho vivo, da produção do cuidado ${ }^{10}$ - objeto dessa pesquisa. Os ruídos funcionam como interrogações da lógica do processo de coordenação na produção do cuidado. Podem ser identificados no decorrer da análise das trajetórias sentinelas ou outros dispositivos analisadores, utilizados para a análise molecular da produção de cuidado ${ }^{10}$.

A quebra do silêncio do cotidiano desvela a presença de processos instituintes que não haviam sido pensados pelo modelo de organização e gestão do equipamento institucional, bem como abre possibilidades de interrogações sobre a maneira como se opera o processo de trabalho e o sentido de suas ações naquele equipamento ${ }^{11}$. Do mesmo modo que no fluxograma, por meio das entrevistas podem-se também evidenciar ou confirmar ruídos analisadores que possibilitaram a exposição da micropolítica do trabalho ${ }^{10}$.

Foi analisada a trajetória sentinela de quatro crianças menores de seis anos. A escolha dos casos foi feita em conjunto com a equipe da unidade. As entrevistas com as mães foram realizadas após a análise dos prontuários. A forma mais antiga e mais difundida de coleta de dados orais é a entrevista. Esta supõe uma conversação continuada entre informante e pesquisador, e o tema sobre o que versa é escolhido por este último, por convir com seu trabalho. Assim, o pesquisador é quem dirige a entrevista, orientando o informante para discorrer sobre o tema ${ }^{12}$. A entrevista não é apenas um trabalho de coleta de dados, mas também um momento de interação entre entrevistador e entrevistado. Tal movimento do entrevistado com o entrevistador não representa risco de comprometimento da objetividade ou falha, mas uma condição de aprofundamento de uma relação intersubjetiva "e é condição sine qua non do êxito da pesquisa qualitativa"13.

Através de duas dinâmicas de grupo, foi proposto como tema de discussão o processo de trabalho em saúde na unidade de saúde investigada, tendo a pediatria como foco central, e identificação dos ruídos que emergissem da prática cotidiana e que, no dia-a-dia, interferissem na qualidade da assistência prestada.

Na dinâmica individual foi solicitado aos participantes que elegessem um ator privilegiado, considerado central. Vários atores foram identificados pelos participantes como elemento 
central; o setor de pediatria, porém, foi destacado pela grande maioria. Após terem eleito um setor central, os participantes da dinâmica individual elegeram diversos setores/atores, tanto da unidade básica de saúde quanto outros setores/unidades de saúde externos, que tinham alguma relação com o setor central escolhido. Assim, diversos setores foram elencados, sendo a enfermagem e a nutrição os que mais se repetiram.

Na dinâmica em grupo, os participantes se subdividiram formando 3 grupos com 4 componentes cada um. Nessa etapa foi solicitado que cada grupo selecionasse três atores considerados estrategicamente importantes e com os quais o setor de pediatria desenvolvesse trabalho rotineiro (setores, categorias profissionais ou outros). Para os setores/categorias profissionais selecionados foi solicitado que cada grupo estabelecesse a rede de petições e compromissos envolvida nessas relações. A partir da rede de petições e compromissos deveriam ser destacados ruídos identificados (os malentendidos, problemas, insatisfações, dificuldades de comunicação que dificultam ou interferem no serviço prestado).

\section{RESULTADOS}

\section{As relações estabelecidas entre os prestadores de cuidado no espaço micropolítico de uma unidade básica de saúde}

A realização do grupo focal permitiu o levantamento e discussão dos ruídos que interferem na assistência prestada através da pediatria e a construção da rede de contratualidade, favorecendo a discussão do grupo acerca do papel e inserção do setor, das relações e da gestão do cuidado. Para constituição do grupo focal foram convidados, sem determinar um teto no número de participantes, os profissionais do setor de pediatria e de setores relacionados a ela. Participaram dessa etapa da pesquisa representantes de todas as categorias profissionais que operam/colaboram na assistência prestada através desse setor específico. Dos 12 participantes do grupo, estiveram presentes: uma médica (pediatra), duas dentistas, uma fonoaudióloga, uma farmacêutica, uma nutricionista, duas acadêmicas de nutrição, três enfermeiras e um técnico administrativo responsável pelo setor de matrícula.

Na discussão de um modo geral, o grupo deu ênfase aos pontos identificados como fracos. Dois aspectos foram citados tanto como pontos fortes quanto como pontos fracos pelo grupo: atendimento individualizado e interação com um setor específico da unidade.

Os setores que mais apareceram se relacionando com 0 setor da pediatria foram a enfermagem, a farmácia, a fonoaudiologia, a odontologia e a psicologia. Através das ferramentas utilizadas foi possível identificar algumas falhas e "insuficiências" no trabalho. Dentre os problemas identificados, foi dado destaque à necessidade de discutir melhor a rede de contratualidade entre a pediatria e 0 arquivo/matrícula. Apesar do destaque sempre presente de que a organização da unidade é centrada no usuário, este foi "esquecido" pelos grupos na análise das relações e do processo de trabalho.

A avaliação final apontou que o grupo focal não foi tão produtivo quanto se esperava, visto que o tempo reservado para sua realização foi curto, além da participação de profissionais do próprio setor da pediatria ter se mostrado muito restrita. As agendas dos profissionais, comprometida com a marcação prévia, representaram uma restrição na composição dos grupos de discussão. A maioria dos profissionais que participou do trabalho proposto pertencia a setores que tinham alguma relação com a pediatria, o que é de certa forma também um ponto positivo. Ainda assim, a adesão entre profissionais que trabalham diretamente ligados à pediatria foi considerada baixa, o que parece gerar um olhar muito particular daqueles que não têm uma relação direta com 0 setor.

Os participantes mostraram-se dispostos a dar continuidade às discussões, confirmando suas presenças na data agendada para apresentação e discussão do estudo.

\section{Ruídos identificados no processo de trabalho dos profissionais em uma unidade básica de saúde - Ruídos identificados no grupo focal}

Um instrumento que se mostrou interessante no processo de desencadeamento da discussão foi a construção das redes de contratualidades entre a pediatria e os outros setores.. Na discussão entre os grupos foram identificados três tipos de ruídos: Ruídos Específicos, Ruídos Intra-institucionais e Ruídos Interpessoais. Não foram identificados Ruídos Interinstitucionais.

Foram denominados específicos os ruídos relacionados aos setores de Matrícula (dificuldade de abertura de prontuários; carência quantitativa de profissionais) e de Farmácia (dificuldades com as letras ilegíveis nos receituários médicos; falta de medicamentos; hora de almoço sem profissionais no setor). Isso porque esses setores foram citados em mais de um grupo no decorrer do grupo focal, além de terem sido notado ruídos em comum dentre os que foram citados pelos grupos em questão.

Foram considerados intra-institucionais os ruídos relativos à Unidade: falta de estrutura para atender a demanda da população; déficit de profissionais médicos e outros; aumento da demanda em pediatria e em outros setores; demora na marcação e resultado (entrega) dos exames, além do extravio dos resultados, e ruído interpessoal aquele que, de certa forma, envolve relacionamentos/comunicação entre profissionais/ setores: falta de inter-relação (comunicação) entre a pediatria e o serviço social da unidade.

\section{Ruídos identificados no Fluxograma}

Apesar de terem sido estudados quatro casos, de um modo geral observa-se que ocorre convergência entre os ruídos identificados. Foram identificados no fluxograma 16 ruídos, a saber: R1 - Patologia não solucionada ou agravamento de quadro; R2 - Ausência de identificação (carimbo/rubrica/ 
assinatura); R3 - Ausência à consulta marcada; R4 Medicação não disponível na farmácia; R5 - Reinfecções constantes de escabiose; R6 - Encaminhamento não confirmado; R7 - Informações incompletas em registro; R8 Longo período para entrega de resultado de exame; R9 Ausência de solicitação de exames; R10 - Patologias dermatológicas repetitivas; R11-Ausência de resultados de exames; R12 - Agravamento de quadro devido à medicação; R13 - Reinfecção parasitária; R14 - Identificação tardia de patologia; R15 - Automedicação e R16 - Longo período para realização de procedimento cirúrgico.
A partir das informações colhidas através da entrevista semi-estrutura com a mãe e a análise de prontuário do paciente, foi elaborado um fluxograma analisador que apresenta integralmente a trajetória da criança desde a primeira vez que foi atendida na unidade de saúde estudada. Parte desse fluxograma está apresentado na Figura 1. No trecho selecionado é possível identificar alguns dos citados: patologia não solucionada ou agravamento do quadro; ausência de identificação do atendente (carimbo/rubrica/assinatura); ausência à consulta marcada; medicação não disponível na farmácia e longo período para entrega de resultado de exame.



Fonte: Fluxograma desenhado a partir das informações coletadas da história pregressa através do prontuário do usuário G.S.M., desde a sua primeira consulta na unidade básica.

\section{Legenda:}

Otorrino: Otorrinolaringologista

UB: Unidade básica onde foi realizada a pesquisa

CR: Consulta de revisão

CD: Conduta

HD: Hipótese diagnóstica

PM: Prescrição médica

QP: Queixa principal
AM: Achado médico

SM: Seio Materno

(-): Ausência de sintoma

HM: Hospital Municipal

EAS: Exame de urina

Odonto: Odontologia

ON: Obstrução nasal

IRA: Infecção respiratória aguda 


\section{ANÁLISE DOS RUÍDOS}

Dos ruídos identificados através do fluxograma analisador alguns também foram relacionados pelos profissionais de saúde da unidade e pela família das crianças (usuários): falta de agilidade dos serviços de apoio diagnóstico para realização dos exames e liberação dos resultados; falta de medicamentos na farmácia; dificuldade de abertura de prontuários e marcação de consultas; dificuldade em compreender o que está escrito nos receituários e prontuários em virtude da ilegibilidade dos mesmos.

Destaca-se a fragilidade da rede de contratualidade estabelecida no interior da unidade estudada, sem que fossem estabelecidos os necessários acordos quanto aos pedidos e compromissos necessários às relações entre os setores. Essa fragilidade na contratualização entre os diversos setores não é específica dessa unidade básica de saúde, pelo contrário, tem sido uma característica comum encontrada em várias outras Unidades Básicas de Saúde. Faz-se necessário o reconhecimento de que essas contratualidades são construídas no dia-a-dia dos múltiplos atores envolvidos, de forma espontânea, dinâmica, extremamente dependente das relações interpessoais existentes e das redes informais de comunicação $0^{10}$.

Alguns pontos não mencionados pelos grupos, e extremamente relevantes na identificação e resolução de problemas, merecem ser analisados mais detidamente já que estes estão diretamente relacionados ao processo de trabalho e com a qualidade da assistência prestada. Durante as entrevistas, algumas mães deram destaque à diferença no acolhimento entre os pediatras. Ainda que se considere que cada profissional aborda o usuário de uma forma muito próxima, há queixas que devem ser consideradas. 0 responsável pela criança informa que não há um critério de prioridades para a entrada na unidade de saúde, o que vem dificultar a remarcação de consulta para o serviço de odontologia. Dos ruídos identificados pela equipe, alguns vêm iluminar a análise sobre o processo de trabalho em saúde: necessidade de recursos humanos (pediatras e profissionais administrativos), falta de estrutura para atender a demanda da população e número e variedade de exames oferecidos disponibilizados pela unidade de saúde; baixa resolubilidade, levando ao agravamento do quadro da criança; ausência de identificação (carimbo, rubrica ou assinatura); recorrência de infecções, ausência de confirmação dos encaminhamentos; registros incompletos, automedicação, falta de data nos prontuários e desordem cronológica das consultas.

Por uma análise inicial acerca das falhas expostas, a partir dos ruídos evidenciados, há algumas tensões no processo de trabalho em saúde na unidade básica de saúde estudada, o que corrobora fortemente para a necessidade de rever o processo de trabalho em saúde nessa área específica.

Apesar dos ruídos destacados, a qualidade da assistência prestada no geral, não parece estar comprometida. Destacase na fala dos usuários uma grande satisfação em relação à qualidade da assistência. Esse fato marca a necessidade de que profissionais e gestores mantenham-se empenhados na solução de problemas que, de certa forma, interferem na qualidade da assistência prestada.

\section{CONSIDERAÇÕES FINAIS}

A análise desses ruídos revelou a fragilidade da rede de contratualidade estabelecida no interior dessa unidade, sem que fossem estabelecidos os necessários acordos quanto aos pedidos e compromissos necessários às relações entre os setores. Faz-se necessário o reconhecimento de que essas contratualidades são construídas no dia-a-dia dos múltiplos atores envolvidos, de forma espontânea, dinâmica, extremamente dependente das relações interpessoais existentes e das redes informais de comunicação. Para tanto é necessário ultrapassar barreiras, especialmente aquelas referentes ao campo das relações. Nesse sentido, a equipe da unidade básica de saúde em questão tem revelado uma grande disposição em explicitar essas tensões e rever tais contratualidades.

Por fim, os ruídos evidenciados nesta análise apontam para tensões no processo de trabalho em saúde na unidade básica de saúde estudada. Essas "falhas" fazem ver a necessidade de rever o processo de trabalho em saúde nessa área específica. Apesar desses ruídos, a qualidade da assistência prestada, no geral, não parece estar comprometida, visto que o usuário entrevistado demonstra satisfação quanto aos serviços oferecidos, apesar dos problemas que este apresenta.

0 estudo viabilizou a identificação dos problemas que interferem no atendimento do setor de pediatria da unidade básica e que, conseqüentemente, afetam a qualidade da assistência prestada. A possibilidade de renovar a parceria ensino-serviço, estabelecida entre a EEAP e a unidade básica de saúde em questão, através da participação de profissionais da rede de saúde, graduandos e professores, tem permitido abrir as "caixas pretas" de um projeto em permanente construção.

\section{Referências}

1) Merhy EE. A perda da dimensão cuidadora na promoção da saúde: uma discussão do modelo assistencial e da intervenção no seu modo de trabalhar a assistência. In: Merhy EE,Campos CR, Malta DC, organizadores. Sistema Único de Saúde em Belo Horizonte: reescrevendo o público. São Paulo (SP): Xamã; 1998.p.103-20.

2) Merhy EE, Onocko R, organizadores . Agir em saúde: um desafio para o público. São Paulo (SP)/ Buenos Aires(AR): Hucitec/Lugar Editorial;1997.

3) Merhy EE. O SUS e um dos seus dilemas: mudar a gestão e a lógica do processo de trabalho em saúde -um ensaio sobre a micropolítica do trabalho vivo. In: Fleury S, organizador. Saúde e democracia: a luta do CEBES. São Paulo (SP): Lemos Ed; 1997a. p.125-142.

4) Silva DC, Alvim NAT, Figueiredo PA. Tecnologias leves em saúde e sua relação com o cuidado de enfermagem hospitalar. Esc Anna Nery Rev Enferm 2008 jun; 12 (2): 291- 99.

5) Baremblitt GF. Compêndio de análise institucional e outras correntes: teoria e prática. Rio de Janeiro (RJ): Rosa dos Tempos; 1996.

6) Minayo MCS. Pesquisa social: teoria, método e criatividade. $16^{\mathrm{a}}$ ed. Petrópolis (RJ): Vozes; 2000. 
7) Hartz ZMA, organizador. Avaliação em saúde: dos modelos conceituais à prática na análise da implantação de programas. Rio de Janeiro (RJ): FIOCRUZ; $1997.131 \mathrm{p}$.

8) Pereira MJB. 0 trabalho da enfermeira no Serviço de Assistência Domiciliar: potência para (re) construção da prática da saúde e de enfermagem. [tese doutrado]. Ribeirão Preto (SP): Escola de Enfermagem /USP; 2001.

9) Gonçalves RBM. Tecnologia e organização social das práticas de saúde: características tecnológicas de processo de trabalho na rede estadual de centros de saúde de São Paulo (SP): Hucitec/ABRASCO; 1994. 278 p.
10) Jorge AO. A gestão hospitalar sob a perspectiva da micropolítica do trabalho vivo. [tese de doutorado] Campinas (SP): Faculdade de Ciências Médicas/UNICAMP; 2002.

11) Flores F. Inventando la empresa del siglo XXI. Santiago(CH): Hachete; 1989.

12) Queiroz MIP. Relatos orais: do "indizível" ao "dizível". In: Cienc\& Cult 1987: 3 ( 39): 272- 86.

13) Minayo MCS. 0 desafio do conhecimento: pesquisa qualitativa em saúde. $7^{a}$ ed. São Paulo (SP): Hucitec; 2000. 